\title{
Outage Performance of Cognitive Relay Networks with Wireless Information and Power Transfer
}

\author{
Zheng Yang, Student Member, IEEE, Zhiguo Ding, Member, IEEE, Pingzhi Fan, Fellow, IEEE, \\ and George K. Karagiannidis, Fellow, IEEE
}

\begin{abstract}
In this paper, we consider an underlay cognitive radio $(C R)$ networks with one primary receiver, one cognitive transmitter-receiver pair, and one energy harvesting relay. The transmission power of the secondary source is determined opportunistically by its interference to the primary receiver, and the relay transmission is powered by the energy harvested from the radio frequency observations at the relay. For the considered CR networks with simultaneous wireless information and power transfer (SWIPT), we derive analytical expressions for the outage probability, as well as their high signal-to-noise ratio (SNR) approximations in closed-form. The developed analytical results demonstrate that the use of SWIPT will not cause any loss of diversity gain, but the outage probability achieved by the SWIPTCR scheme asymptotically decays as $\frac{\log S N R}{S N R}$, whereas a decaying rate of $\frac{1}{S N R}$ is achieved by a conventional CR network. Computer simulation results are also provided to demonstrate the accuracy of the presented analysis.
\end{abstract}

Index Terms-Cognitive radio systems, decode-and-forward, simultaneous wireless information and power transfer, energy harvesting.

\section{INTRODUCTION}

Cooperative cognitive radio networks is a promising method to improve the spectrum utilization efficiency, and has attracted considerable attention during the last years. The outage probability and the asymptotic diversity gain of cooperative cognitive radio systems, based on the decode-and-forward (DF) strategy, have been studied in [1], while the performance of cognitive relaying networks with the primary user's outage constrain has been investigated in [2].

Recently, energy harvesting has received significant attention, since it is a promising technology to prolong the lifetime of energy constrained wireless networks. The application of energy harvesting to cognitive radio (CR) networks has been studied in [3]. The performance of a cognitive metro-cellular network using solar power, was derived in [4]. The throughput of an energy harvesting CR system with a slotted mode, has been investigated in [5], where the secondary user is

Z. Yang and P. Fan are with the Institute of Mobile Communications, Southwest Jiaotong University, Chengdu 610031, P. R. China. (email: zyfjnu@163.com, p.fan@ieee.org).

Z. Ding is with the School of Computing and Communications, Lancaster University, LA1 4YW, UK. (e-mail: z.ding@lancaster.ac.uk).

G. K. Karagiannidis is with the ECE Department, Aristotle University of Thessaloniki, Thessaloniki, Greece and with the ECE Department, Khalifa University, Abu Dhabi, UAE. (e-mail: geokarag@auth.gr).

The work of Z. Yang and P. Fan was supported by the 973 Program (No. 2012CB316100), NSFC Project (No. 61471302), the 111 Project (No. 1112-14). The work of Z. Ding was supported by the UK EPSRC under grant number EP/I037423/1. powered by the energy harvested from natural energy sources, while the throughput maximization for the secondary user with finite battery capacity has been studied in [6]. Furthermore, the throughput maximization for secondary users has also been studied in [7], when the secondary users perform radio frequency energy harvesting as well as reuse the spectrum of the primary network simultaneously. Very recently, the authors [8] considered energy harvesting in a large scale noncooperative $\mathrm{CR}$ network, which contains multiple cognitive transmitter-receiver pairs and one primary transmitter-receiver pair. More specifically, energy harvesting is performed at secondary receivers. However, most existing energy harvesting solutions rely on natural energy sources, such as wind and solar power [9], and the recent developed concept, simultaneous wireless information and power transfer (SWIPT) can realize energy harvesting in more demanding indoor environments [10]. It has been shown that SWIPT has the potential to reduce the energy consumption at wireless nodes, particularly at the relays, [11], [12]. In [13], the authors investigated the robust power minimization problem for the multiple input single output (MISO) downlink scenario, with a multi-antenna SWIPT relay. The performance of an energy harvesting amplify-andforward relay with multiple antennas, have been studied in [14]. Furthermore, in [15] and [16], the authors studied the outage performance of energy harvesting in cooperative $\mathrm{CR}$ networks, where the secondary transmitter and the relay are powered by the energy harvested from the signals transmitted by the primary user.

The aim of this paper is to study the performance of the cooperative cognitive radio system, where the cognitive transmitter communicates with the cognitive receiver via an energy harvesting relay. In particular, the transmission power of the secondary source is determined opportunistically to ensure that the interference at the primary receiver does not exceed a predetermined threshold. On the other hand, the relay transmission is powered by the energy harvesting from the signals sent by the secondary transmitter. Because the relay transmission power is coupled with the source-relay channel condition, the calculation of the outage probability for the addressed wireless power transfer system is much more challenging compared to that in conventional energy harvesting relaying systems. Specifically, we first obtain an exact analytical expression for the outage probability of the transmitter-receiver pair in cognitive relay networks, where the relay is powered by the energy harvested from the cognitive transmitter. Then, asymptotic studies are carried out to show that the outage performance decays as $\frac{\log S N R}{S N R}$, if the maxi- 
mum interference constraint at the primary user is proportional to the maximum cognitive transmission power, where SNR denotes the signal-to-noise ratio. Note that for the cognitive radio network under consideration, a much faster decaying rate of $\frac{1}{S N R}$ can be achieved if the relay uses its own battery to power the relay transmission. Finally, more asymptotic studies are carried out by focusing on cases with different choices for the interference constraint at the primary receiver and the secondary transmitter power.

\section{SYSTEM MODEL}

We consider a cooperative $\mathrm{CR}$ networks which contains a primary receiver, a cognitive transmitter-receiver pair and one energy harvesting relay. It is assumed that all of nodes are equipped with a single antenna. The cognitive transmitter communicates with its destination via the secondary relay. All the channels are assumed to be subjected independent and identically (i.i.d.) Rayleigh fading. The channel gains from the cognitive transmitter to the primary receiver and the relay are denoted by $X_{s p}$ and $X_{s r}$, respectively. While those from the relay to the primary receiver and the destination as $X_{r p}$ and $X_{r d}$. It is also assumed that the secondary transmitter and relay have the perfect channel state information (CSI). Furthermore, it is assumed, as in [1], [17] that there is no direct link between the cognitive transmitter and the cognitive receiver, and the primary transmitter is located far away from the secondary relay and the cognitive destination, and thus it does not cause any interference to them.

The interference power at the primary user should not exceed the maximum tolerable level $I$, and therefore the cognitive transmitter power should satisfy

$$
P_{s}=\min \left\{P, \frac{I}{X_{s p}}\right\},
$$

where $P$ is the maximum secondary transmission power.

During the first time slot, the cognitive transmitter sends a message, $x$, to the relay. Note that the energy harvested at the relay will be used only as relay transmission power, and other energy consumption to support the transmitter/receiver circuits and the information detection circuits has not been considered in this paper. Therefore, the observation used for information detection is given by

$$
y_{r}=x \sqrt{P_{s}(1-\rho) X_{s r}}+w,
$$

where $0 \leq \rho \leq 1$ is the power splitting factor [10], and $w$ is the additive white Gaussian noise (AWGN), denoted by $w \sim$ $\mathcal{C N}(0,1)$.

Provided that the relay can successfully detect the message from the cognitive transmitter, i.e.,

$$
\frac{1}{2} \log \left(1+P_{s}(1-\rho) X_{s r}\right) \geq R,
$$

the power splitting factor needs to satisfy the following constraint, $\rho \leq 1-\frac{\varepsilon}{P_{s} X_{s r}}$, where $\varepsilon=2^{2 R}-1$ and $R$ denotes the targeted data rate. Since all of the left power will be utilized for energy harvesting [10], [11], the optimal value of the power splitting factor is $\rho_{o}=1-\frac{\varepsilon}{P_{s} X_{s r}}$. Therefore the transmission power obtained at the relay after energy harvesting is given by

$$
P_{r}^{\prime}=\eta P_{s} X_{s r} \rho_{o}= \begin{cases}\eta\left(P_{s} X_{s r}-\varepsilon\right), & \text { if } P_{s} X_{s r}>\varepsilon \\ 0, & \text { otherwise }\end{cases}
$$

where $0<\eta \leq 1$ denotes the energy harvesting efficiency [10].

Similar to the cognitive transmitter, the interference power caused by the relay to the primary user cannot exceed $I$. Therefore, the relay power should satisfy

$$
P_{r}=\min \left\{P_{r}^{\prime}, \frac{I}{X_{r p}}\right\} .
$$

During the second time slot, the cognitive DF relay forwards the decoded message to the cognitive receiver with the transmission power $P_{r}$, if the message is correctly decoded at the relay in the first time slot.

\section{OUTAGE PERFORMANCE OF THE ENERGY HARVESTING SYSTEM}

The end-to-end outage probability with the DF relay is given by

$\mathrm{P}_{\text {out }}=\underbrace{\operatorname{Pr}\left\{P_{s} X_{s r}<\varepsilon\right\}}_{Q_{1}}+\underbrace{\operatorname{Pr}\left\{P_{s} X_{s r}>\varepsilon, P_{r} X_{r d}<\varepsilon\right\}}_{Q_{2}}$.

An analytical expression for the outage probability can be obtained as in the following Theorem.

Theorem 1: The outage probability of the energy harvesting in $\mathrm{CR}$ cooperative networks is given by

$$
\begin{aligned}
\mathrm{P}_{\text {out }}= & 1+\left(\frac{\varepsilon}{\varepsilon+I} e^{-\frac{I+\varepsilon}{P}}-e^{-\frac{\varepsilon}{P}}\right)\left(g(0)-\frac{\varepsilon}{\varepsilon+I} g(I)\right) \\
& +\frac{2 \varepsilon}{\eta(\varepsilon+I)}(g(y, 0)-g(y, I))
\end{aligned}
$$

where

$$
\begin{gathered}
g(j)=2 \sqrt{\frac{\varepsilon+j}{\eta P}} \mathbf{K}_{1}\left(2 \sqrt{\frac{\varepsilon+j}{\eta P}}\right), \\
g(y, j)=\int_{\frac{I}{P}}^{\infty} \mathbf{K}_{0}\left(2 \sqrt{\frac{y(\varepsilon+j)}{\eta I}}\right) e^{-\frac{I+\varepsilon}{I} y} d y,
\end{gathered}
$$

$j=0, I$ and $\mathbf{K}_{n}(\cdot)$ denotes the modified Bessel function of the second kind [20, eq. (3.324.1)].

Proof: See Appendix A.

The analytical expressions derived in Theorem 1 can be easily used to evaluate the outage probability numerically, but it does not provide much insight into the impact of the channel and system parameters, which motivates the asymptotic study in the following Corollary. In order to find the diversity gain, we assume that the power $P$ tends to infinity.

Corollary 1: When $I$ is proportional to $P$, i.e. $I=v P$, and $P \rightarrow \infty$, the outage probability decays as

$$
\mathrm{P}_{\mathrm{out}}=\frac{\log P}{P}+O\left[\left(\frac{\log P}{P}\right)^{2}\right] .
$$

Proof: See Appendix B. 
Note that the condition, $I=v P$, means that the effect of the interference is non-negligible, even if the cognitive transmitter increases its transmission power to the infinity [1], [17]. The above Corollary shows that a diversity gain equal to one is still achievable, although the relay does not use its own battery for powering relay transmissions. However, as shown at the end of this section, the decaying rate shown in Corollary 1 is quite small. Therefore an important question to be answered is what is the reason for such a small decaying rate. Note that the above analytical results are developed based on the assumption that there is a constraint for the secondary transmission power. In the following we will study the performance when the cognitive transmitter has unlimited transmission power i.e, $\bar{P}_{s}=\frac{I}{X_{s p}}$, which will indicate the impact of the cognitive transmission constraint.

Theorem 2: When the cognitive transmitter has unlimited transmission power, then the outage probability is given by

$$
\begin{aligned}
\overline{\mathrm{P}}_{\text {out }}= & \frac{2 \varepsilon}{I+\varepsilon}-\frac{\varepsilon^{2}}{(I+\varepsilon)^{2}}+\frac{I \varepsilon}{(I+\varepsilon)^{2} \eta} \\
& \times\left(e^{\frac{\varepsilon}{(I+\varepsilon) \eta}} \boldsymbol{E}_{1}\left(\frac{\varepsilon}{(I+\varepsilon) \eta}\right)-e^{\frac{1}{\eta}} \boldsymbol{E}_{1}\left(\frac{1}{\eta}\right)\right),
\end{aligned}
$$

where $\boldsymbol{E}_{1}(x)=\int_{x}^{\infty} \frac{e^{-t}}{t} d t$ is the exponential integral [18, eq. (5.1.1)].

Proof: The result can be obtained from (7) straightforwardly. When the cognitive transmitter has unlimited transmission power, this means $P \rightarrow \infty$ in Theorem 1 . Let $t=\frac{I+\varepsilon}{I} y$, $g(y, j)$ in (7) can be rewritten as

$$
\begin{aligned}
g(y, j) & =\frac{I}{I+\varepsilon} \int_{0}^{\infty} \mathbf{K}_{0}\left(2 \sqrt{\frac{\varepsilon+j}{(\varepsilon+I) \eta} t}\right) e^{-t} d t \\
& =\frac{1}{2} \frac{I}{I+\varepsilon} e^{\frac{\varepsilon+j}{(\varepsilon+I) \eta}} \boldsymbol{E}_{1}\left(\frac{\varepsilon+j}{(\varepsilon+I) \eta}\right),
\end{aligned}
$$

where the last equation follows from [19, eq. (11)].

Substituting (12) and $P \rightarrow \infty$ into (7), the proof is completed.

Corollary 2: When the cognitive transmitter has unlimited transmission power and $I \rightarrow \infty$, then the asymptotic outage probability is given by

$$
\overline{\mathrm{P}}_{\mathrm{out}}=\frac{\log I}{I}+O\left[\left(\frac{\log I}{I}\right)^{2}\right] .
$$

Proof: When $x \rightarrow 0$, the exponential integral $\boldsymbol{E}_{1}(x)$ can be approximated as [18, eq. (5.1.11)]

$$
\begin{aligned}
\boldsymbol{E}_{1}(x) & =-\gamma-\ln x-\sum_{n=1}^{\infty} \frac{(-1)^{n} x^{n}}{n n !} \\
& =\ln \frac{1}{x}+O\left[\left(\ln \frac{1}{x}\right)^{2}\right],
\end{aligned}
$$

where $\gamma$ is the Euler's constant.

The Corollary is proved by substituting (14) into Theorem 2.

From Corollary 1 and Corollary 2, it is interesting to observe that the asymptotic expressions of the outage probabilities for both cases share the same structure.

\section{A. Cognitive Radio Networks with a Conventional Relay}

In a conventional cooperative cognitive network, holds that $P_{s}=\min \left\{P, \frac{I}{X_{s p}}\right\}$, and the relay power is not a function of the source-relay channel condition, i.e., $P_{r}^{0}=\min \left\{P, \frac{I}{X_{r p}}\right\}$. Based on (6), the outage probability of the cognitive relay network, denoted by $P_{0}$, can be expressed as

$$
\mathrm{P}_{0}=Q_{1}+\left(1-Q_{1}\right) \operatorname{Pr}\left\{P_{r}^{0} X_{r d}<\varepsilon\right\} .
$$

Since all the channels are i.i.d., the outage probability $\operatorname{Pr}\left\{P_{r}^{0} X_{r d}<\varepsilon\right\}$ is the same as $Q_{1}=\operatorname{Pr}\left\{P_{s} X_{s r}<\varepsilon\right\}$ in (19). Thus, when $I=v P$, and $P \rightarrow \infty$, the outage probability $P_{0}$ can be expressed as

$$
\begin{aligned}
\mathrm{P}_{0} & =2\left(1-e^{-\frac{\varepsilon}{P}}+\frac{\varepsilon e^{-\frac{I+\varepsilon}{P}}}{I+\varepsilon}\right)-\left(1-e^{-\frac{\varepsilon}{P}}+\frac{\varepsilon e^{-\frac{I+\varepsilon}{P}}}{I+\varepsilon}\right)^{2} \\
& =\frac{1}{P}+O\left[\frac{1}{P^{2}}\right] .
\end{aligned}
$$

When the cognitive transmit power is much higher than $\frac{I}{X_{s p}}$, i.e, $\bar{P}_{s}=\frac{I}{X_{s p}}$, and $I \rightarrow \infty$, similarly to (16), the asymptotic outage probability, denoted by $\bar{P}_{0}$, is given by

$$
\overline{\mathrm{P}}_{0}=\frac{1}{I}+O\left[\frac{1}{I^{2}}\right]
$$

Comparing Corollary 1 and Corollary 2 to (16) and (17), one can conclude that the outage probability of the energy harvesting system has a slower decaying rate, and this is mainly due to the fact that the relay does not use its own battery to power relay transmissions. However, it is worthy pointing out that both cognitive systems achieve the same diversity gain, which can be explained briefly as follows. Because of the use of SWIPT, the relay transmission power is $\eta\left(P_{s} X_{s r}-\varepsilon\right)$, instead of $P$ as in conventional cooperative networks. Recall that the diversity order is obtained when letting $P \rightarrow \infty$, and the relaying power difference between the cases with and without SWIPT becomes insignificant when $P \rightarrow \infty$. Therefore, the diversity order achieved by the two schemes should be the same.

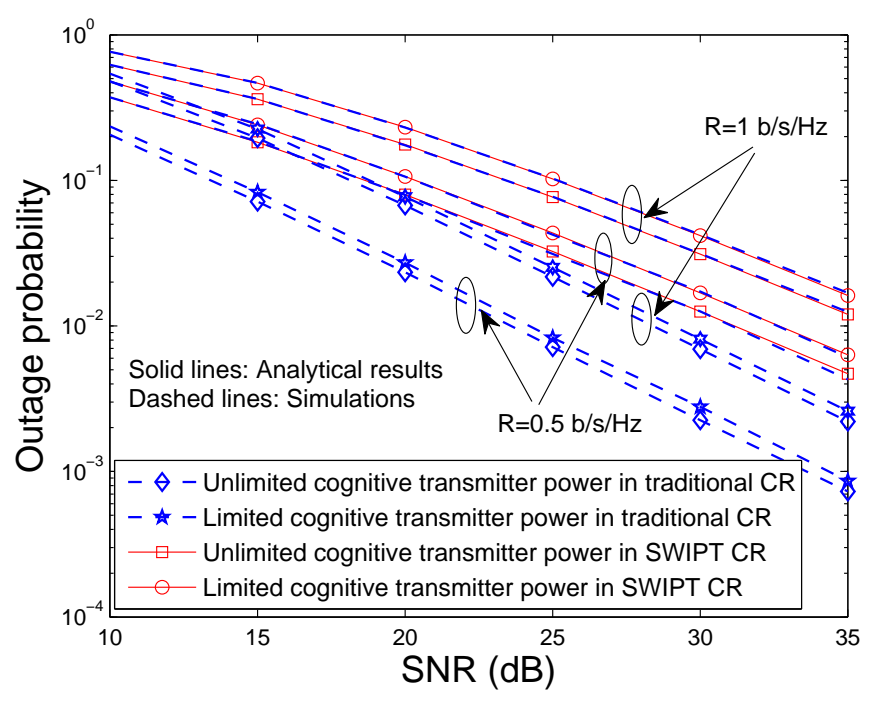

Fig. 1. Analytical results vs Monte Carlo simulations, $I=P, \eta=$ 0.5 . 


\section{Simulations AND Discussion}

In this section, we verify our theoretical results via Monte Carlo simulations. Fig. 1 shows that the analytical results obtained in Theorem 1 and Theorem 2 match well with Monte Carlo simulations, and both of two schemes achieve a diversity gain of one. This is because when the tolerable interference level at the primary receiver, $I$, is proportional to the maximum power level $P$, then $I$ can be replaced by $v P$. Furthermore, the performance for the case without the cognitive transmission power constraint is better than that of the case with the constraint. The reason is that the cognitive transmitter power for the case with the constraint, $P_{s}=\min \left\{P, \frac{I}{X_{s p}}\right\}$, is less than that of the other without the constraint, $P_{s}=\frac{I}{X_{s p}}$. It can also be seen from Fig. 1 that the outage probability of cognitive relay networks with SWIPT is larger than the conventional cognitive relay networks, since the relay does not use its own battery to power relaying transmissions. In addition, all the outage curves in Fig. 1 are parallel to each other at the high SNR region, which means cognitive relay networks with SWIPT will not lose the diversity gain compared with conventional cognitive relay networks.

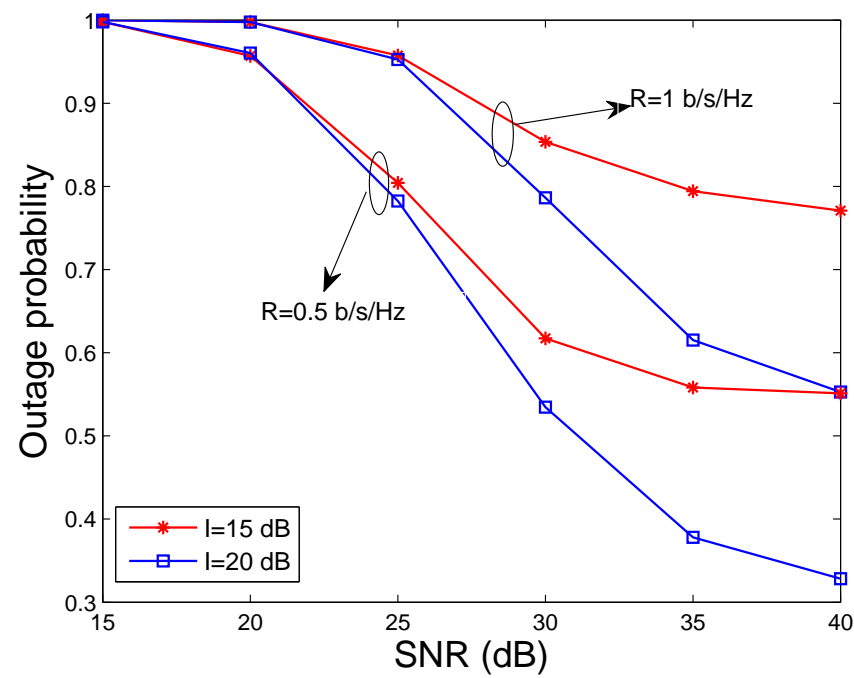

Fig. 2. The impact of the interference power constraint $I$ on the outage probability, $\eta=0.3$. Assume that path loss factor $\alpha=2$, cognitive transmitter, relay, secondary user, and primary user are located at coordinates $(0,0),(2,2),(5,0)$, and $(3,3)$, respectively.

Fig. 2, shows the impact of the predetermined interference threshold at the primary receiver on the performance of the cognitive users. As it can be seen from Fig. 2, when the interference threshold $I$ is not scaling proportionally to $P$, the interference constraint will significantly affect the performance of the cognitive receiver, and an outage probability floor appears in the figure, which means that no diversity gain is achievable. This can be explained as follows. For a fixed interference threshold $I$, increasing $P$ does not necessarily increase the cognitive transmitter power, $P_{s}$, which is capped at $\min \left\{P, \frac{I}{X_{s p}}\right\}$. For example, consider an extreme case with $P \rightarrow \infty$, and one can find that $P_{s}=\frac{I}{X_{s p}}$, which is no longer a function of SNR.

\section{Conclusions}

In this paper, the outage performance of cooperative CR networks with an energy harvesting relay has been studied. The developed analytical results demonstrate that the use of SWIPT deteriorate outage performance; however, a diversity gain of one is still achievable in CR networks with SWIPT, the same as conventional CR networks. In this paper, a scenario with one primary user pair has been considered, and it is an important future direction to study the scenario with multiple user pairs, where game theory is ideal to be used for modelling user interaction. Furthermore, we can conclude that it is also important to apply multiple input multiple output to the addressed SWIPT-CR networks for further performance improvement.

\section{APPENDIX A}

\section{Proof of THEOREM 1}

Conditioned on $P_{s}=\min \left\{P, \frac{I}{X_{s p}}\right\}$, the outage probability $Q_{1}=\operatorname{Pr}\left\{P_{s} X_{s r}<\varepsilon\right\}$ in (6) can be expressed as

$$
Q_{1}=\operatorname{Pr}\left\{X_{s r}<\frac{\varepsilon}{P}, X_{s p}<\frac{I}{P}\right\}+\underbrace{\operatorname{Pr}\left\{\frac{X_{s r}}{X_{s p}}<\frac{\varepsilon}{I}, X_{s p}>\frac{I}{P}\right.}_{Q_{11}}\} .
$$

It is easy to obtain

$$
\operatorname{Pr}\left\{X_{s r}<\frac{\varepsilon}{P}, X_{s p}<\frac{I}{P}\right\}=\left(1-e^{-\frac{\varepsilon}{P}}\right)\left(1-e^{-\frac{I}{P}}\right),
$$

while $Q_{11}$ can be evaluated as

$$
Q_{11}=\int_{\frac{I}{P}}^{\infty}\left(1-e^{-\frac{\varepsilon x}{I}}\right) e^{-x} d x=e^{-\frac{I}{P}}-\frac{I}{I+\varepsilon} e^{-\frac{I+\varepsilon}{P}} .
$$

Based on $P_{r}=\min \left\{P_{r}^{\prime}, \frac{I}{X_{r p}}\right\}$ in (5), $Q_{2}$ in (6) can be expressed as

$$
\begin{aligned}
Q_{2} & =\operatorname{Pr}\left\{P_{s} X_{s r}>\varepsilon, P_{r} X_{r d}<\varepsilon\right\} \\
& =\operatorname{Pr}\left\{P_{s} X_{s r}>\varepsilon, P_{r}^{\prime} X_{r d}<\varepsilon, P_{r}^{\prime}<\frac{I}{X_{r p}}\right\} \\
& +\operatorname{Pr}\left\{P_{s} X_{s r}>\varepsilon, \frac{I}{X_{r p}} X_{r d}<\varepsilon, P_{r}^{\prime}>\frac{I}{X_{r p}}\right\}
\end{aligned}
$$


Since $P_{r}^{\prime}=\eta\left(P_{s} X_{s r}-\varepsilon\right)$, and $P_{s}=\min \left\{P, \frac{I}{X_{s p}}\right\}, Q_{2}$ can be further rewritten in (18), at the bottom of the previous page.

The four terms $Q_{2 i}$ will be evaluated respectively as

$$
\begin{aligned}
Q_{21}= & \int_{\frac{\varepsilon}{P}}^{\infty} F_{X_{r d}}\left(\frac{\varepsilon}{\eta(P x-\varepsilon)}\right) F_{X_{r p}}\left(\frac{I}{\eta(P x-\varepsilon)}\right) f_{X_{s r}}(x) d x \\
= & \frac{e^{-\frac{\varepsilon}{P}}}{P} \int_{0}^{\infty}\left(1-e^{-\frac{\varepsilon}{\eta t}}\right)\left(1-e^{-\frac{I}{\eta t}}\right) e^{-\frac{t}{P}} d t \\
= & e^{-\frac{\varepsilon}{P}}\left(1-\sqrt{\frac{4 I}{\eta P}} \mathbf{K}_{1}\left(\sqrt{\frac{4 I}{\eta P}}\right)-\sqrt{\frac{4 \varepsilon}{\eta P}} \mathbf{K}_{1}\left(\sqrt{\frac{4 \varepsilon}{\eta P}}\right)\right. \\
& \left.+2 \sqrt{\frac{\varepsilon+I}{\eta P}} \mathbf{K}_{1}\left(2 \sqrt{\frac{\varepsilon+I}{\eta P}}\right)\right),
\end{aligned}
$$

where the second equation follows from $t=P x-\varepsilon$, and the last one is obtained by using the definition of $\mathbf{K}_{n}(\cdot)$ in [20, eq. (3.324.1)].

Similarly, $Q_{22}$ can be obtained as

$$
\begin{aligned}
Q_{22}= & e^{-\frac{\varepsilon}{P}}\left(\sqrt{\frac{4 I}{\eta P}} \mathbf{K}_{1}\left(\sqrt{\frac{4 I}{\eta P}}\right)\right. \\
& \left.-\frac{2 I}{\varepsilon+I} \sqrt{\frac{\varepsilon+I}{\eta P}} \mathbf{K}_{1}\left(2 \sqrt{\frac{\varepsilon+I}{\eta P}}\right)\right) .
\end{aligned}
$$

While $Q_{23}$ can be rewritten as

$$
\begin{aligned}
Q_{23}= & \int_{\frac{I}{P}}^{\infty} \int_{\frac{\varepsilon}{I} y}^{\infty} F_{X_{r d}}\left(\frac{\varepsilon}{\eta\left(\frac{I}{y} x-\varepsilon\right)}\right) F_{X_{r p}}\left(\frac{I}{\eta\left(\frac{I}{y} x-\varepsilon\right)}\right) \\
& \times f_{X_{s r}}(x) d x f_{X_{s p}}(y) d y \\
= & \int_{\frac{I}{P}}^{\infty} \int_{0}^{\infty} \frac{y}{I}\left(1-e^{-\frac{\varepsilon}{\eta s}}\right)\left(1-e^{-\frac{I}{\eta s}}\right) e^{-\frac{y s}{I}} d s e^{-\frac{I+\varepsilon}{I} y} d y \\
= & \frac{I}{\varepsilon+I} e^{-\frac{\varepsilon+I}{P}}-\left(h_{1}(y)+h_{1}(y, 0)-h_{1}(y, \varepsilon)\right), \quad(25)
\end{aligned}
$$

where the second equation follows from $s=\frac{I}{y} x-\varepsilon$, and

$$
h_{1}(y)=\int_{\frac{I}{P}}^{\infty} 2 \sqrt{\frac{y}{\eta I}} \mathbf{K}_{1}\left(2 \sqrt{\frac{y}{\eta I}}\right) e^{-\frac{I+\varepsilon}{I} y} d y
$$

$$
h_{1}(y, j)=\int_{\frac{I}{P}}^{\infty} 2 \sqrt{\frac{y(\varepsilon+j)}{\eta I}} \mathbf{K}_{1}\left(2 \sqrt{\frac{y(\varepsilon+j)}{\eta I}}\right) e^{-\frac{I+\varepsilon}{I} y} d y,
$$

$j=0, I$.

Similar to $Q_{23}$, the result of $Q_{24}$ is given by

$$
Q_{24}=h_{1}(y)-\frac{I}{I+\varepsilon} h_{1}(y, \varepsilon) .
$$

Since the derivation of $x^{v} \mathbf{K}_{v}(x)$ is given by $\left(x^{v} \mathbf{K}_{v}(x)\right)^{\prime}=$ $-x^{v} \mathbf{K}_{v-1}(x)$, thus the derivation of

$$
K_{1}(y, j) \triangleq \sqrt{\frac{4 y(\varepsilon+j)}{\eta I}} \mathbf{K}_{1}\left(\sqrt{\frac{4 y(\varepsilon+j)}{\eta I}}\right)
$$

in $h_{1}(y, j)$ can be given as

$$
K_{1}^{\prime}(y, j)=-2(\varepsilon+j) \mathbf{K}_{0}\left(\sqrt{\frac{4 y(\varepsilon+j)}{\eta I}}\right) /(\eta I) .
$$

By using partial integration, the integral $h_{1}(y, j)$ in $(25)$ and (28) can be rewritten as

$$
h_{1}(y, j)=\frac{I e^{-\frac{I+\varepsilon}{P}}}{I+\varepsilon} g(j)-\frac{2(\varepsilon+j)}{\eta(I+\varepsilon)} g(y, j),
$$

where $g(j)$ and $g(y, j)$ are defined in (7), $j=0, I$.

Substituting (20-21), (23-28), (31) and $\operatorname{Pr}\left\{X_{s p}<\frac{I}{P}\right\}=$ $1-e^{\frac{I}{P}}$ into (6), the proof is completed.

\section{APPENDIX B}

\section{ProOF OF COROLlaRY 1}

By applying the series representation of Bessel function $\mathbf{K}_{0}(z)$ [20, eq. (8.447)]

$$
\mathbf{K}_{0}(z)=-\ln \left(\frac{z}{2}\right) \sum_{k=0}^{\infty} \frac{\left(\frac{z}{2}\right)^{2 k}}{(k !)^{2}}+\sum_{k=0}^{\infty} \frac{z^{2 k}}{2^{2 k}(k !)^{2}} \psi(k+1) \text {. }
$$

Thus, $K_{0}(y, j) \triangleq \mathbf{K}_{0}\left(2 \sqrt{\frac{y(\varepsilon+j)}{\eta I}}\right)$ in

$$
g(y, j)=\int_{\frac{I}{P}}^{\infty} \mathbf{K}_{0}\left(2 \sqrt{\frac{y(\varepsilon+j)}{\eta I}}\right) e^{-\frac{I+\varepsilon}{I} y} d y
$$

in (7) can be rewritten as

$$
\begin{aligned}
K_{0}(y, j) & =\sum_{k=0}^{\infty} \frac{y^{k}\left(\frac{\varepsilon+j}{\eta I}\right)^{k}}{(k !)^{2}}(\psi(k+1) \\
& \left.-\ln \left(\frac{\varepsilon+j}{\eta I}\right)^{\frac{1}{2}}-\ln y^{\frac{1}{2}}\right) .
\end{aligned}
$$

The expression $K_{0}(y, j)$ contains two different variable $y^{k}$ and $y^{k} \ln y$, which means we need to calculate

$$
Q_{3}=\int_{\frac{I}{P}}^{\infty} y^{k} e^{-\frac{I+\varepsilon}{I} y} d y
$$

and

$$
Q_{4}=\int_{\frac{I}{P}}^{\infty} \ln (y) y^{k} e^{-\frac{I+\varepsilon}{I} y} d y
$$

in $g(y, j)$ in (7). It is easy to obtain $Q_{3}$ as

$$
Q_{3}=\left(\frac{I}{I+\varepsilon}\right)^{k+1} \Gamma\left(k+1, \frac{I+\varepsilon}{P}\right) \approx \Gamma(k+1, v),
$$

where $\Gamma(s, x)=\int_{x}^{\infty} t^{s-1} e^{-t} d t$ is an upper incomplete function. Let $t=\frac{I+\varepsilon}{I} y, Q_{4}$ can be rewritten as

$$
Q_{4}=\left(\frac{I}{I+\varepsilon}\right)^{k+1} \int_{\frac{I+\varepsilon}{P}}^{\infty} \ln (t) t^{k} e^{-t} d t \triangleq\left(\frac{I}{I+\varepsilon}\right)^{k+1} Q_{5} .
$$

Since $\int t^{k} e^{-t} d t=-e^{-t} \sum_{i=0}^{k} \frac{k !}{(k-i) !} t^{k-i}$, we can use partial integration to calculate $Q_{5}$ as

$$
\begin{aligned}
Q_{5}= & \ln \left(\frac{I+\varepsilon}{P}\right) e^{-\frac{I+\varepsilon}{P}} \sum_{i=0}^{k} \frac{k !}{(k-i) !}\left(\frac{I+\varepsilon}{P}\right)^{k-i} \\
& +\sum_{i=0}^{k-1} \frac{k !}{(k-i) !} \Gamma\left(k-i, \frac{I+\varepsilon}{P}\right)+\boldsymbol{E}_{1}\left(\frac{I+\varepsilon}{P}\right) \\
\approx & \ln v e^{-v} \sum_{i=0}^{k} \frac{k ! v^{k-i}}{(k-i) !}+\sum_{i=0}^{k-1} \frac{k ! \Gamma(k-i, v)}{(k-i) !}+\boldsymbol{E}_{1}(v) \\
\triangleq & G(i, k) .
\end{aligned}
$$


Substituting (32-37), and (38) into $g(y, j)$, we can obtain the asymptotic expression of $g(y, j)$ as

$$
g(y, 0) \approx \ln I
$$

and

$$
g(y, I) \approx \frac{2}{\eta} \sum_{k=0}^{\infty} \frac{\Gamma(k+1, v) \psi(k+1)}{(k !)^{2}}-\frac{1}{\eta} \sum_{k=0}^{\infty} \frac{G(i, k)}{(k !)^{2}},
$$

which is a constant. Recall that when $x \rightarrow 0, x \mathbf{K}_{1}(x) \approx$ $1+\frac{x^{2}}{2} \ln \left(\frac{x}{2}\right)[20$, eq. (8.446)]. Thus,

$$
g(0)=2 \sqrt{\frac{\varepsilon}{\eta P}} \mathbf{K}_{1}\left(2 \sqrt{\frac{\varepsilon}{\eta P}}\right) \approx 1+\frac{\varepsilon}{\eta P} \ln \frac{\varepsilon}{\eta P}=1 .
$$

Substituting (39-40), and (41) into (7), the proof is completed.

\section{REFERENCES}

[1] K. Kim, T. Duong, and H. Poor, "Outage probability of single-carrier cooperative spectrum sharing systems with decode-and-forward relaying and selection combining," IEEE Trans. Wireless Commun., vol. 12, pp. 806-417, Feb. 2013.

[2] J. Si, Z. Li, X. Chen, B. Hao, and Z. Liu, "On the performance of cognitive relay networks under primary users outage constrain," IEEE Commun. Lett., vol. 15, pp. 422-424, Apr. 2011.

[3] S. Lee, R. Zhang, and K. Huang, "Opportunistic wireless energy harvesting in cognitive radio networks," IEEE Trans. Wireless Commun., vol. 12, no. 9, pp. 4788-4799, July 2013.

[4] S. Zaidi, M. Ghogho, D. McLernon, and A. Swami, "Energy harvesting empowered cognitive metro-cellular networks," in Proc. International Workshop on Cognitive Cellular Systems (CCS), Sept. 2014, pp. 1-5.

[5] S. Yin, Z. Qu, and S. Li, "Achievable throughput optimization in energy harvesting cognitive radio systems," IEEE Journal on Selected Areas in Commun., vol. 33, no. 3, pp. 407-422, Mar. 2015.

[6] J. Pradha, S. Kalamkar, and A. Banerjee, "Energy harvesting cognitive radio with channel-aware sensing strategy," IEEE Commun. Lett., vol. 18, no. 7, pp. 1171-1174, July 2014 .

[7] S. Lee, K. Huang, and R. Zhang, "Cognitive energy harvesting and transmission from a network perspective," in Proc. IEEE International Conference on Communication Systems (ICCS), Nov. 2012, pp. 225-229.

[8] L. Sibomana, H. Zepernick, and H. Tran, "Wireless information and power transfer in an underlay cognitive radio network," in Proc. International Conference on Signal Processing and Communication Systems (ICSPCS), Dec. 2014, pp. 1-7.

[9] S. Park, H. J. Kim, and D. Hong, "Cognitive radio networks with energy harvesting," IEEE Trans. Wireless Commun., vol. 12, no. 3, pp. 13861397, Mar. 2013.

[10] X. Zhou, R. Zhang, and C. Ho, "Wireless information and power transfer: Architecture design and rate-energy tradeoff," IEEE Trans. Commun., vol. 61, no. 11, pp. 4754-4767, Nov. 2013.

[11] Z. Ding, S. Perlaza, I. Esnaola, and H. Poor, "Power allocation strategies in energy harvesting wireless cooperative networks," IEEE Trans. Wireless Commun., vol. 13, pp. 846-460, Feb. 2014.

[12] A. Nasir, X. Zhou, S. Durrani, and R. Kennedy, "Relaying protocols for wireless energy harvesting and information processing," IEEE Trans. Wireless Commun., vol. 12, no. 7, pp. 3622-3636, July 2013.

[13] Y. Yuan, Z. Chu, Z. Ding, K. Cumanan, M. Johnston, "Joint relay beamforming and power splitting ratio optimization in a multi-antenna relay network," in Proc. IEEE Wireless Communications and Signal Processing (WCSP), Oct. 2014, pp. 1-5.

[14] G. Zhu, C. Zhong, H. Suraweera, G. Karagiannidis, Z. Zhang, T. Tsiftsis, "Wireless information and power transfer in relay systems with multiple antennas and interference," IEEE Trans. Commun., vol: PP, no. 99, DOI: 10.1109/TCOMM.2015.2398862.

[15] S. Mousavifar, Y. Liu, C. Leung, M. Elkashlan, and T. Duong, "Wireless energy harvesting and spectrum sharing in cognitive radio," in Proc. IEEE Vehicular Technology Conference (VTC), Sept. 2014, pp. 1-5.

[16] Z. Wang, Z. Chen, L. Luo, Z. Hu, B. Xia, and H. Liu, "Outage analysis of cognitive relay networks with energy harvesting and information transfer," in Proc. IEEE International Conference on Communications (ICC), June 2014, pp. 4348-4353.
[17] J. Lee, H. Wang, J. G. Andrews, and D. Hong, "Outage probability of cognitive relay networks with interference constraints," IEEE Trans. Wireless Commun., vol. 10, no. 2, pp. 390-395, Feb. 2011.

[18] M. Abramowitz and I. Stegun, Handbook of Mathematical Functions: with Formulas, Graphs, and Mathematical Tables, No. 55. Courier Corporation, 1964.

[19] M. Geller and E. W. Ng, "A table of integrals of the exponential integral," Journal of Research of the National Bureau of Standards, vol. 73B, no. 3, pp. 191-210, Sept. 1969.

[20] I. S. Gradshteyn and I. M. Ryzhik, Table of Integrals, Series and Products, 6th ed., New York: Academic Press, 2000. 